\title{
"SÁCALE LOS PIES AL MOSQUITO»: RESULTADOS PARCIALES DE LA IMPLEMENTACIÓN DE UN PROGRAMA EDUCATIVO EN REPÚBLICA DOMINICANA ${ }^{1}$
}

\section{"Sácale los pies al mosquito»: partial results of the implementation of an educational programme in the Dominican Republic}

\section{Yohan Enmanuel Vásquez Bautista}

Estudiante de Educación y Asistente de Investigación del Laboratorio de Entomología, Universidad Agroforestal Fernando Arturo de Meriño (UAFAM). Jarabacoa, República Dominicana

Correo-e: a.investigacion2@uafam.edu.do

\section{Yisel Hernández Barrios}

Investigadora y Profesora del Departamento Control de Vectores, Instituto de Medicina Tropical Pedro Kourí (IPK). La Habana, Cuba. Correo-e: yhbarrios@ipk.sld.cu

\section{María Altagracia Rodríguez Sosa}

Estudiante de Agronomía y Asistente de Investigación del Laboratorio de Entomología, Universidad Agroforestal Fernando Arturo de Meriño (UAFAM). Jarabacoa, República Dominicana. Correo-e: a.investigacion1@uafam.edu.do

\section{Elizabeth del Carmen Rosario}

Estudiante de Educación y Técnico del Laboratorio de Entomología, Universidad Agroforestal Fernando Arturo de Merińo (UAFAM). Jarabacoa, República Dominicana Correo-e: elizabethrosario00809@gmail.com

\section{Jesús Confesor Durán Tiburcio}

Estudiante de Agronomía y Técnico del Laboratorio de Entomología, Universidad Agroforestal Fernando Arturo de Meriño (UAFAM). Jarabacoa, República Dominicana Correo-e: jsduran675@gmail.com

\section{Pedro María Alarcón-Elbal}

Investigador y Profesor del Laboratorio de Entomología, Universidad Agroforestal Fernando Arturo de Meriño (UAFAM). Jarabacoa, República Dominicana. Autor para correspondencia: Correo-e: pedro.alarcon@uv.es

Recibido: 5/4/2019 • Aprobado: 2/6/2019

Cómo citar: Vásquez Bautista, Y. E., Hernández Barrios, Y., Rodríguez Sosa, M. A., del Carmen Rosario, E., Durán Tiburcio, J. C., \& Alarcón-Elbal, P. M. (2019). «Sácale los pies al mosquito»: resultados parciales de la implementación de un programa educativo en República Dominicana. Ciencia Y Sociedad, 44(3), 33-49. https://doi.org/10.22206/cys.2019.v44i3.pp33-49

\section{Resumen}

En República Dominicana, las campañas de concientización encaminadas a informar, sensibilizar y movilizar a las comunidades en la eliminación de los riesgos asociados a la presencia de enfermedades vehiculadas por mosquitos (Diptera: Culicidae) se desarrollan, fundamentalmente, durante los períodos epidémicos, careciendo de sostenibilidad en el tiempo. El objetivo del presente trabajo fue desarrollar el programa educativo «Sácale los pies al mosquito» para la prevención y el control de enfermedades vehiculadas por mosquitos en Jarabacoa, municipio ubicado en la Cordillera Central. El programa consta de cuatro fases: diagnóstico, planeación, ejecución y evaluación. En la fase de diagnóstico se aplicó una encuesta CAP a 17 centros educativos

\begin{abstract}
In the Dominican Republic, awareness campaigns aimed at informing, sensitizing and mobilizing communities to eliminate the risks associated with the presence of mosquito-borne diseases (Diptera: Culicidae) take place mainly during epidemic periods, with no sustainability over time. The objective of this work was to develop the educational program «Sácale los pies al mosquito» for the prevention and control of mosquito-borne diseases in Jarabacoa, a municipality located in the Cordillera Central. The programme consists of four phases: diagnosis, planning, implementation and evaluation. In the diagnostic phase, a KAP survey was applied to 17 secondary schools, for a total of 639 respondents. Based on the results obtained, educational
\end{abstract}

1. Este trabajo forma parte del proyecto de investigación "Sistema integrado de educación y vigilancia entomológica para la prevención y el control de enfermedades vehiculizadas por mosquitos (Diptera: Culicidae) en dos polos turísticos de República Dominicana", subvencionado por FONDOCyT / MESCyT. Convocatoria 2015: Proyecto No. 2015-112-145. 
de enseñanza media, para un total de 639 encuestados. A partir de los resultados obtenidos se desarrollaron charlas educativas y talleres dirigidos a satisfacer las necesidades de aprendizaje identificadas y fomentar la participación consciente y sostenible de los escolares en las acciones de prevención y control, tanto en el entorno escolar como comunitario. Conjuntamente, se creó un sitio web como espacio de divulgación científica sobre las temáticas abordadas. Los resultados obtenidos, a partir de la implementación del programa educativo, han aportado elementos claves para promover la participación de las comunidades en la prevención y control de esta problemática, así como garantizar la sostenibilidad de las acciones desarrolladas.

Palabras clave: programa de enseñanza; encuesta CAP; mosquitos; arbovirosis; República Dominicana.

\section{Introducción}

Las enfermedades vehiculadas por vectores representan una amenaza permanente para la salud global, por su distribución, incidencia y elevadas tasas de morbilidad y mortalidad. Debido a la diversidad de factores que inciden en la emergencia y reemergencia de las mismas, se han convertido en objeto de estudio y campo de actuación profesional de diversas disciplinas, evidenciándose cada vez más la necesidad de asumir enfoques integrados para su prevención y control (WHO, 2012; OMS, 2017). Sin embargo, a pesar de los avances en este campo y del respaldo de organismos internacionales, las características geoclimáticas, poblacionales, económicas y sociales de la región del Caribe han contribuido históricamente a crear marcos favorables para su transmisión (Alarcón-Elbal et al., 2017). De estas enfermedades, las arbovirosis, vehiculadas por el mosquito Aedes aegypti (Linnaeus, 1762), fundamentalmente, han tenido especial connotación en los últimos años; entre ellas: el dengue, el chikungunya, el Zika y la fiebre amarilla (OPS, 2016).

Una mirada al escenario epidemiológico de República Dominicana en relación con estas enfermedades demuestra la importancia de adecuar las acciones tradicionales de prevención y control, dando talks and workshops were developed aimed at satisfying the identified learning needs and promoting the conscious and sustainable participation of school children in prevention and control actions, both in the school and community environment. Together, a website was created as a space for scientific dissemination on the topics addressed. The results obtained from the implementation of the educational program have provided key elements to promote the optimal participation of communities in the prevention and control of this problem, as well as ensuring the sustainability of the actions developed.

Keywords: teaching program; KAP survey; mosquitoes; arbovirosis; Dominican Republic.

lugar a iniciativas contemporáneas. La malaria y el dengue, por ser endémicas en el país, acaparan el principal foco de atención, pero también deben tenerse en cuenta otras como el chikungunya y el Zika, de aparición reciente en La Española. Estas enfermedades constituyen un serio problema de salud pública ya que presentan un patrón de transmisión prácticamente ininterrumpido durante todo el año, convirtiéndose en un freno para el desarrollo económico y social (Alarcón-Elbal et al., 2017). Esta problemática está lejos de desaparecer, como se infiere de la situación epidemiológica reciente; de hecho, hasta la Semana Epidemiológica 25 se han notificado 3,433 casos, incluyendo tres fallecimientos auditados por el Comité de Auditoría Clínica, con una elevada incidencia en menores de quince años (MSP/DIGEPI, 2019).

De manera tradicional, la lucha contra los culícidos y los patógenos que estos transmiten ha conducido, inevitablemente, a la reducción de sus criaderos y al reordenamiento ambiental (Schweigmann et al., 2009). Sin embargo, en el caso concreto de Ae. aegypti y Aedes albopictus (Skuse, 1894), se postula como necesaria, imprescindible e impostergable la participación comunitaria o ciudadana (Diéguez Fernández, Sosa Cabrera y Pérez Arruti, 2013), ya que se trata de insectos sinantrópicos y, por tanto, 
estrechamente vinculados a las viviendas humanas. Las estrategias para movilizar a todos los sectores de la sociedad son esenciales, siendo necesario incidir en la promoción del trabajo colectivo y el desarrollo de redes de apoyo para alcanzar una acción transformadora en los miembros de la comunidad y lograr así un mayor compromiso social (Sánchez et al., 2008). Para ello, resulta esencial desarrollar intervenciones que partan del diagnóstico de los conocimientos, actitudes y prácticas de las poblaciones estudiadas. Aunque se ha demostrado que los conocimientos adecuados sobre estas enfermedades y sus vectores no necesariamente conducen a cambios de comportamientos (San Martín \& Prado, 2004), resulta esencial conocer estas variables para satisfacer las necesidades de aprendizaje, promover una actitud positiva y responsable, así como fomentar buenas prácticas de prevención y control que se correspondan con las acciones promovidas por organismos internacionales e iniciativas nacionales afines. Además, la incorporación de los escolares en las actividades educativas, como multiplicadores de la información en su entorno familiar, y en las actividades de inspección y eliminación de los criaderos en las viviendas, ha demostrado ser muy efectiva (Sánchez Valdés, 2006).

Unido a lo anterior, organismos internacionales y nacionales promueven el desarrollo de acciones de educación y comunicación que contribuyen a fomentar la participación consciente, activa y sostenida de las comunidades en la prevención y el control de los vectores de importancia médica y las enfermedades vehiculadas por estos (WHO, 2012, OPS, 2016; OMS, 2017). Asimismo, subsiste la necesidad de poner los resultados investigativos a disposición de la población, mediante un lenguaje sencillo, oportuno, asequible y contextualizado a las peculiaridades de los diferentes sectores de la sociedad. Por consiguiente, entre los principales desafíos de las investigaciones sociales se encuentran el diseño, la implementación, el monitoreo y la evaluación de iniciativas que permitan fortalecer la integración y participación de diferentes actores sociales; así como facilitar su acceso y colaboración en el desarrollo de las mismas (Hernández, 2017).

En República Dominicana, las campañas de concientización encaminadas a informar, sensibilizar y movilizar a las comunidades en la eliminación de los riesgos asociados a la presencia de enfermedades vehiculadas por mosquitos se desarrollan, fundamentalmente, durante los períodos epidémicos, careciendo de sostenibilidad en el tiempo. Por consiguiente, ante la situación epidemiológica actual y la experiencia acumulada, se revela la necesidad de promover un abanico más amplio de acciones que satisfagan las necesidades de aprendizaje de diferentes actores sociales, contribuyan a su participación consciente y sostenida en las acciones de prevención y control, y se ajusten a las particularidades de cada escenario y momento epidemiológico. En esta línea de actuación, se ha implantado un nuevo programa educativo llamado «Sácale los pies al mosquito» para la prevención y el control de enfermedades vehiculadas por mosquitos en Jarabacoa.

\section{Materiales y métodos}

\section{Diseño general del estudio}

El programa educativo «Sácale los pies al mosquito», desarrollado como resultado del presente estudio, comenzó en 2016 con la misión de trascender el carácter reactivo de las acciones tradicionales para enfocarse en la prevención, prestando especial atención al contexto social y a las particularidades de cada comunidad. El nombre del proyecto nace de la expresión dominicana "sacar los pies", que significa irse, escapar, salir corriendo, dejar a alguien o apartarlo de su lado. Este tuvo como población diana los centros educativos de enseñanza media, teniendo en cuenta la elevada concentración de jóvenes en estas instituciones, la ausencia de intervenciones similares y el reconocimiento de la importancia de los estudiantes como promotores de los conocimientos, las percepciones y las prácticas, y como agentes de cambio en sus escuelas y comunidades de residencia. 
El programa consta de cuatro fases: diagnóstico, planeación, ejecución y evaluación. En la primera fase se aplicó una encuesta de conocimientos, actitudes y prácticas (CAP) sobre la problemática relacionada con los mosquitos y las enfermedades vehiculadas por estos. Los indicadores a explorar se seleccionaron mediante la revisión de la literatura sobre el tema, entrevista a informantes claves y los intercambios del equipo de investigación. El cuestionario incluyó preguntas cerradas, de elecciones múltiples y abiertas. La encuesta fue sometida a la validación por criterio de expertos y posteriormente a una prueba piloto siguiendo la lista de comprobación propuesta por Goode y Hatt (1991) (citado en Casas Anguita, Repullo Labradora \& Donado Campos, 2003). Lo anterior permitió hacer los ajustes necesarios al instrumento para su posterior aplicación (figura 1A). Paralelamente, en esta fase se llevaron a cabo encuestas y levantamientos entomológicos domiciliarios con el objetivo de valorar el nivel de infestación en diferentes barrios del municipio (Diéguez Fernández, Borge de Prada, Rodríguez Sosa, Vásquez Bautista \& Alarcón-Elbal, 2019; Rodríguez Sosa, Diéguez Fernández, Borge de Prada, Vásquez Bautista \& Alarcón-Elbal, 2019).

La fase de planeación permitió, a partir del análisis de los resultados de la encuesta, identificar los destinatarios de la intervención, definir las líneas de mensaje, los recursos necesarios y las estrategias a seguir, y diseñar las acciones concretas a desarrollar, así como el cronograma de las mismas.

En la fase de ejecución se llevaron a cabo "videodebates" (figura 1B), charlas educativas (figura 1C), talleres de capacitación, distribución de productos comunicativos como cartelería informativa (figura 1D) y creación de espacios digitales de divulgación científica. Los contenidos temáticos de estas acciones de educación y comunicación fueron seleccionados por el equipo de investigación a partir de los resultados de la encuesta. De este modo se ajustaron a las necesidades de aprendizaje y a los elementos contextuales de las comunidades de residencia de los escolares y de los centros educacionales incluidos en el estudio.

La cuarta fase, todavía en ejecución, comprende una evaluación continua que abarca desde la etapa de diagnóstico hasta la ejecución del programa, lo que ha permitido hacer los ajustes necesarios. Sin embargo, tres años después de la implementación del programa se realizará una evaluación final que será objeto de otras publicaciones. Para ello se aplicará nuevamente la encuesta inicial, de modo que se pueda, a partir de la comparación de antes y después, cuantificar cambios en los conocimientos, percepciones y prácticas de los estudiantes de enseñanza media de Jarabacoa, así como aportar evidencias del impacto científico y social del proyecto ejecutado. Asimismo, se realizarán nuevos levantamientos entomológicos domiciliarios que se compararán con los iniciales a efectos de explorar las diferencias entre los niveles de infestación antes y después de la implementación del programa.

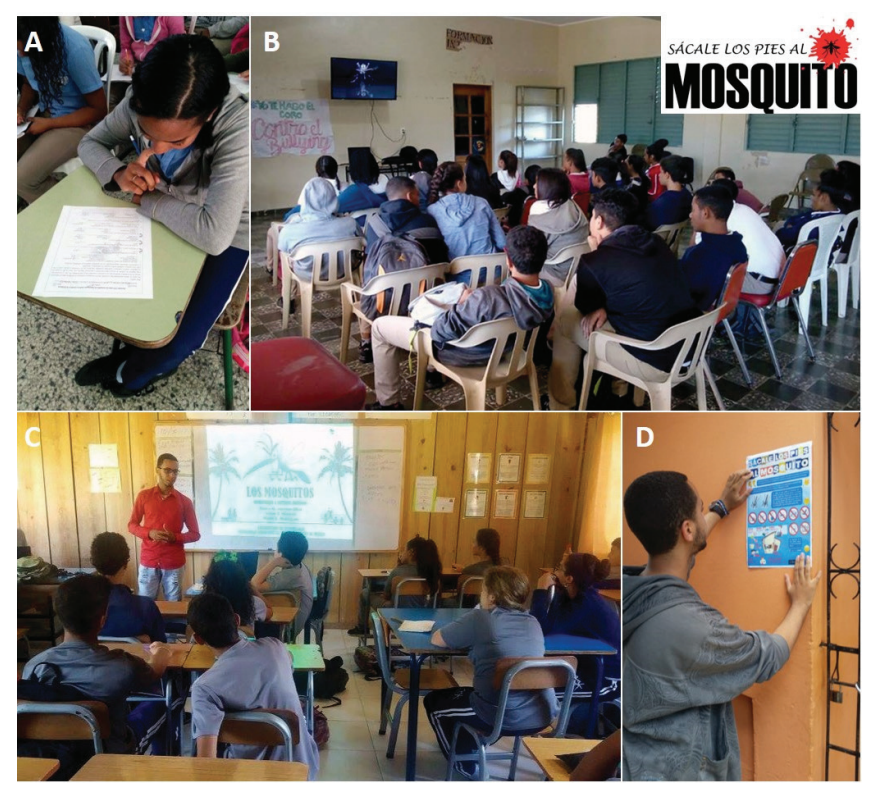

Figura 1. Actividades realizadas en el marco del programa educativo, donde: A) aplicación de encuesta CAP; B) videodebate; C) charlas educativas; D) distribución de productos comunicativos.

Fuente: archivo personal de los autores. 


\section{Selección y caracterización del área de estudio}

El estudio se llevó a cabo en Jarabacoa, República Dominicana, municipio perteneciente a la provincia de La Vega, ubicado en la Cordillera Central, a una altura promedio de $530 \mathrm{msnm}$ y con un área de $23 \mathrm{~km}^{2}$. Esta localidad cibaeńa, conocida por su benévolo clima primaveral, se ha consolidado en los últimos años como un referente ecoturístico en el Caribe gracias a la riqueza natural que alberga (Orgaz Agüera y Cañero Morales, 2015), la cual consta de una población en torno a los setenta mil habitantes. En este territorio se han reportado hasta la fecha dieciséis especies de mosquitos, entre las que se incluyen aquellas de mayor importancia médica, como $A a$. aegypti, Ae. albopictus, Culex quinquefasciatus (Say, 1823) y Anopheles albimanus (Wiedemann, 1820), entre otras (Rodríguez Sosa et al., 2019).

\section{Selección de la muestra}

Los centros educativos se seleccionaron mediante un muestreo por conveniencia, teniendo en cuenta la cercanía entre los mismos, las comunidades de residencia de los escolares y la disponibilidad de los directivos para realizar el estudio. Para la selección posterior de los encuestados se tuvo en cuenta que fueran estudiantes de $6^{\circ}$ curso de Educación Básica o de los niveles de Bachiller $\left(1^{\circ}, 2^{\circ}, 3^{\circ}\right.$ y $4^{\circ}$ ), que perteneciera a uno de los colegios seleccionados y que dieran su consentimiento informado a participar en el estudio. Lo anterior contribuyó a garantizar la heterogeneidad de la muestra, teniendo en cuenta las variables sociodemográficas establecidas en el estudio (edad, sexo, grado, colegio y comunidades de residencia). Es de resaltar que en esta investigación se presentaron algunas limitaciones, entre ellas que no todos los centros educativos consultados consintieron participar en las actividades del programa. Además, los resultados se presentan de forma meramente descriptiva, sin que pueda inferirse significatividad estadística, como sucede en otros similares (Castrillón Castro, Santa Gil, Loaiza Giraldo, Ortiz Garcés \& Aragon Múnera, 2017).

\section{Procesamiento y análisis de la información}

Para el procesamiento y análisis de los datos cuantitativos se utilizó el programa SPSS versión 19.0. Los datos se analizaron utilizando métodos de estadística descriptiva y analítica, teniendo en cuenta las variables e indicadores establecidos. En cuanto al análisis de los datos cualitativos se utilizó el programa Nvivo versión QSR Nvivo10 (QSR International Pty LTD, Melbourne, Australia, 2010). Para ello, se establecieron categorías inductivas y deductivas que facilitaron el análisis y la presentación de los resultados.

\section{Resultados}

En la fase de diagnóstico la muestra quedó conformada por 17 centros educativos de enseńanza media y un total de 639 encuestados provenientes de 43 de los barrios de residencia de Jarabacoa; se apreció un predominio de escolares menores de 15 años y del sexo femenino (tabla 1).

Tabla 1. Características sociodemográficas de los escolares encuestados, Jarabacoa, 2017-2018.

\begin{tabular}{|c|c|c|c|c|c|c|c|c|c|}
\hline \multirow{2}{*}{} & \multicolumn{4}{|c|}{ Edad } & \multicolumn{2}{c|}{ Sexo } & \multicolumn{4}{c|}{ Grado } \\
\cline { 2 - 10 } & $\mathbf{1 0 - 1 2}$ & $\mathbf{1 3 - 1 5}$ & $\mathbf{1 6 - 1 8}$ & $\mathbf{F}$ & $\mathbf{M}$ & $\mathbf{6}$ SEC & $\mathbf{1}^{\circ}$ BACH & 2 BACH & $3^{\circ}$ BACH \\
\hline $\boldsymbol{n}$ & 299 & 278 & 62 & 343 & 296 & 206 & 200 & 137 & 96 \\
\hline$\%$ & 46,8 & 43,5 & 9,7 & 53,7 & 46,3 & 32,2 & 31,3 & 21,4 & 15 \\
\hline
\end{tabular}

Fuente: elaboración propia. 
Con relación a los conocimientos explorados, cabe destacar que la mayoría de los encuestados refirieron conocer sobre los mosquitos de importancia médica $(75,3 \%)$ y las enfermedades vehiculadas por estos $(89,2 \%)$. Sin embargo, se pudo constatar que menos del $10 \%$ mencionaron nombres de las especies y, entre estas, la más referida fue Ae. aegypti. En cuanto a las enfermedades, las arbovirosis resultaron las más conocidas, en tanto el resto fueron identificadas por menos del $20 \%$ de los encuestados. También se mencionaron, aunque en un bajo porcentaje (4,2\%), otras enfermedades que no se corresponden con las exploradas en el estudio (cólera, elefantiasis, gripe), así como síntomas específicos (fiebre, malestar, decaimiento y diarrea, por solo mencionar algunos). Se considera pertinente destacar que solo el $2 \%$ manifestó no conocer ninguna enfermedad.

Al explorar en los síntomas de las enfermedades vehiculadas por mosquitos, los más conocidos fueron la fiebre y el dolor de cabeza, aunque resultó significativo que un elevado porcentaje percibiera que estas enfermedades podían causar la muerte (47,6\%). En relación con los factores de riesgo asociados a la presencia de mosquitos de importancia médica, los más mencionados fueron la falta de higiene, así como la presencia de depósitos con agua y microvertederos. Asimismo, los principales sitios de cría se asociaron a los riesgos antes mencionados, apreciándose que el porcentaje más elevado fueron los basureros (tabla 2).

La principal fuente de información identificada por los escolares fue la televisión. Resultó significativo que la escuela constituyera la segunda, en tanto la comunidad estuvo entre las menos mencionadas. No obstante, solo el $1 \%$ no refirió ninguna (figura 2).
Tabla 2. Conocimientos de los escolares encuestados sobre mosquitos y enfermedades vehiculadas por estos, Jarabacoa, 2017-2018.

\begin{tabular}{|c|c|c|}
\hline Conocimientos explorados & $\mathbf{N}$ & $\%$ \\
\hline \multicolumn{3}{|l|}{ Enfermedades vehiculadas por mosquitos } \\
\hline Dengue & 603 & 94,4 \\
\hline Chikungunya & 594 & 93,0 \\
\hline Encefalitis & 67 & 10,5 \\
\hline Malaria & 113 & 17,7 \\
\hline Fiebre Amarilla & 292 & 45,7 \\
\hline Zika & 521 & 81,5 \\
\hline Filariasis & 47 & 7,4 \\
\hline Otras & 21 & 3,3 \\
\hline No sabe & 12 & 1,9 \\
\hline \multicolumn{3}{|l|}{ Sintomas de las enfermedades } \\
\hline Fiebre & 583 & 91,2 \\
\hline Dolor de cabeza & 529 & 82,8 \\
\hline Erupciones en la piel/Rash & 278 & 43,5 \\
\hline Vómitos & 447 & 70 \\
\hline Dolor muscular/Dolor en las articulaciones & 478 & 74,8 \\
\hline Diarrea & 349 & 54,6 \\
\hline Muerte & 304 & 47,6 \\
\hline Otras & 27 & 4,2 \\
\hline No sabe & 30 & 4,7 \\
\hline \multicolumn{3}{|c|}{ Factores de riesgo asociados a la presencia de mosquitos } \\
\hline Falta de higiene & 401 & 62,8 \\
\hline Ríos/Fosas/Charcos & 371 & 58,1 \\
\hline Vegetación & 66 & 10,3 \\
\hline Depósitos con agua & 401 & 62,8 \\
\hline Características de las viviendas & 132 & 20,7 \\
\hline Otras & 27 & 4,2 \\
\hline Microvertederos & 215 & 33,6 \\
\hline Clima & 105 & 16,4 \\
\hline No sabe & 31 & 4,9 \\
\hline \multicolumn{3}{|l|}{ Sitios de cría } \\
\hline Depósitos de agua limpia & 130 & 20,3 \\
\hline Basureros & 513 & 80,3 \\
\hline Bebederos de animales & 371 & 58,1 \\
\hline Neumáticos & 277 & 43,3 \\
\hline Charcos/Pantanos & 403 & 63,1 \\
\hline Árboles/Axilas de plantas & 112 & 17,5 \\
\hline Botellas/Latas/Vasos & 380 & 59,5 \\
\hline Fosas & 255 & 39,9 \\
\hline Ríos & 274 & 42,9 \\
\hline Vegetación & 118 & 18,5 \\
\hline Otras & 23 & 3,6 \\
\hline No sabe & 22 & 3,4 \\
\hline
\end{tabular}

Fuente: elaboración propia. 


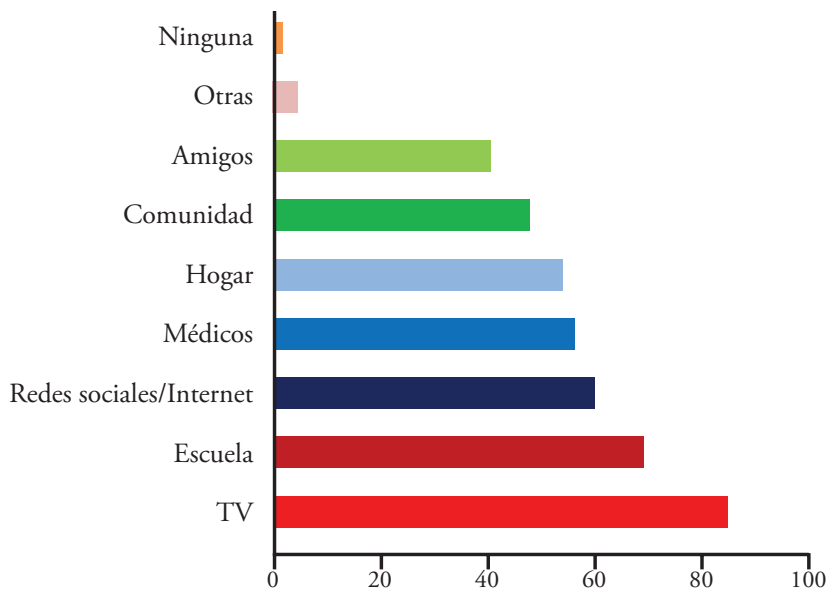

Figura 2. Principales fuentes de información identificadas por los encuestados sobre mosquitos y enfermedades vehiculadas por estos, Jarabacoa, 2017-2018.

Fuente: elaboración propia.

A pesar de las escasas experiencias previas de los encuestados en acciones de prevención y control, en general, y de educación y comunicación, en particular, el 78,7 \% de los escolares manifestó su disposición a participar en las mismas, lo que demuestra un predominio de actitud positiva hacia las acciones que se generaran como parte del proyecto educativo. Otro elemento que refuerza esa actitud positiva es que el $56 \%$ se reconoció como responsable de desarrollar estas acciones, aunque evidenciaron la importancia del abordaje intersectorial de estas; mencionaron al personal de salud (59,5\%), al gobierno $(37,4 \%)$ y a otros sectores de la sociedad (19,6\%). Otro elemento importante es que el $65 \%$ de los encuestados percibieron el riesgo a enfermar. El porcentaje de encuestados que no percibieron el riesgo a enfermar (18\%) o que no saben si se encuentran o no expuestos al riesgo (17\%) se comportó de forma similar.

$\mathrm{Al}$ indagar en las prácticas de prevención y control, se pudo constatar que más del $50 \%$ de los encuestados hicieron referencia a mantener la higiene, tapar los depósitos de agua, fumigar, así como usar repelentes y mosquiteros. También fueron mencionadas otras, aunque en menor proporción $(3,4 \%)$, tales como: destruir criaderos, usar abanicos [ventilador], cloro en el agua o ropa larga. Solo el $4 \%$ de los encuestados no mencionó ninguna (figura 3).

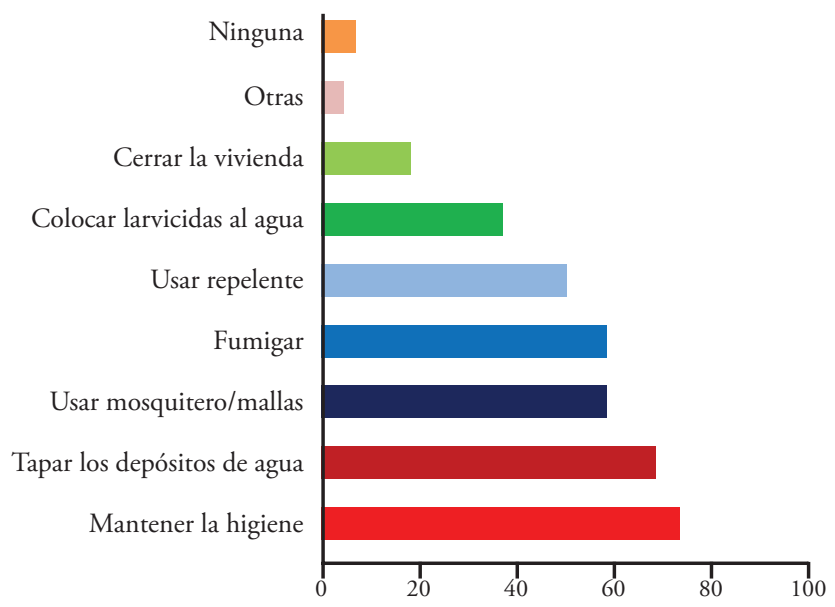

Figura 3. Prácticas de prevención y control referidas por los encuestados sobre mosquitos y enfermedades vehiculadas por estos, Jarabacoa, 2017-2018.

Fuente: elaboración propia.

A pesar de las prácticas antes referidas, la mayoría (71,2 \%) manifestó no haber participado en ninguna iniciativa de educación, comunicación y movilización social sobre estas enfermedades y sus vectores. Además, destacaron que en sus comunidades de residencia prevalecen las iniciativas individuales, resultando insuficientes las colectivas.

En relación con las necesidades de aprendizaje, resultó significativo que solo 24 encuestados no hicieran referencia a ningún contenido. A partir de la codificación de las líneas de mensajes referidas se agruparon en tres categorías fundamentales (prevención y control; ecología; enfermedades), las que a su vez integraban contenidos más específicos. El resto, por su baja frecuencia de aparición y por no ajustarse a ninguna de las categorías antes referidas, se incluyeron en otras. La figura 4 muestra el número de referencia de los contenidos mencionados por los encuestados, nótese que las principales necesidades de aprendizaje estuvieron relacionadas con la ecología de estos vectores. 


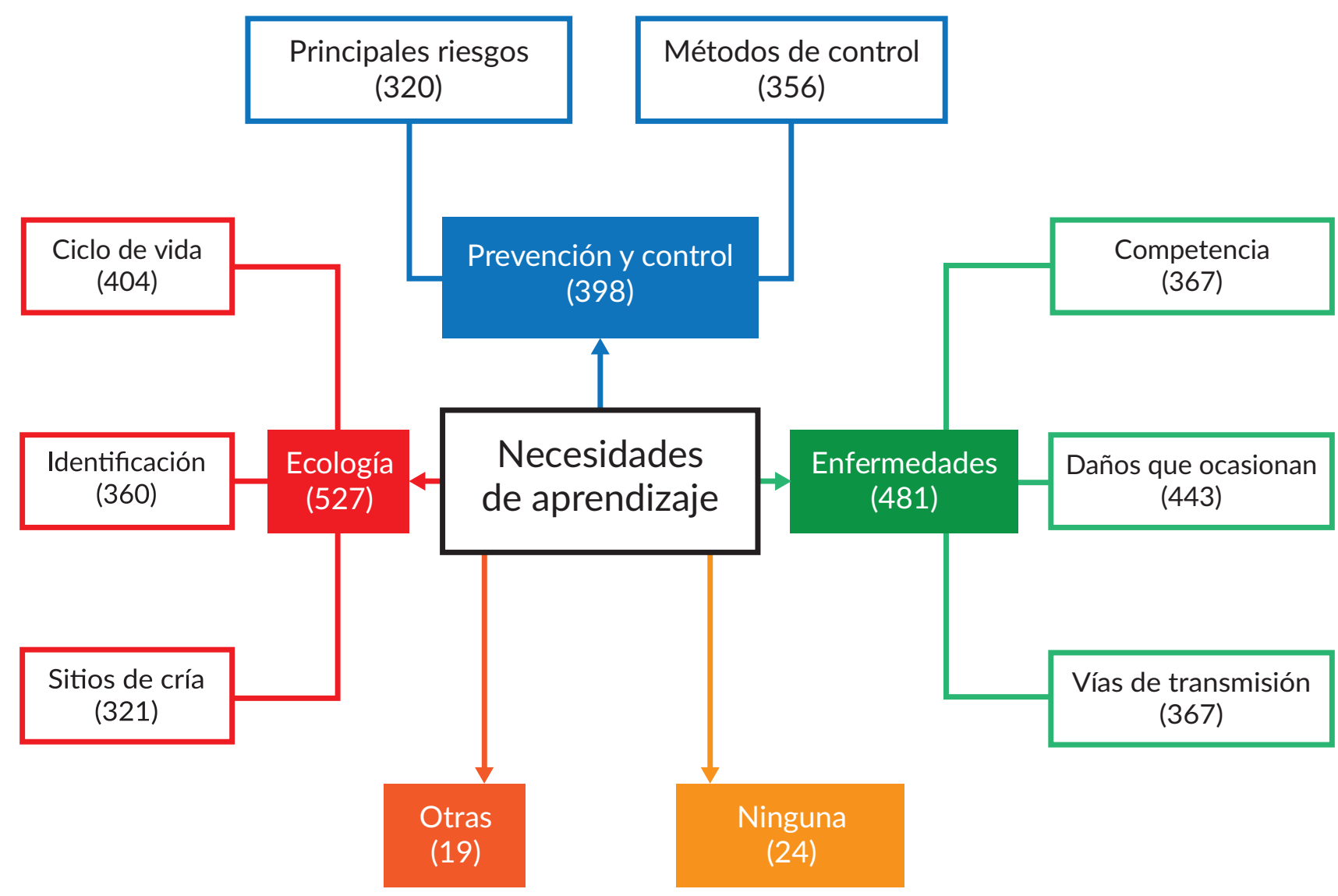

Figura 4. Necesidades de aprendizaje referidas por los encuestados ( $\mathrm{n}=$ cantidad de referencias), sobre mosquitos y enfermedades vehiculadas por estos, Jarabacoa, 2017-2018.

Fuente: elaboración propia.

Las charlas educativas, conversatorios y el uso de las nuevas tecnologías fueron las acciones más recomendadas por los encuestados para satisfacer sus necesidades de aprendizaje, aunque también hicieron referencia al desarrollo de exposiciones, trabajos voluntarios, confección de propagandas y otras acciones más específicas que variaron de acuerdo a las experiencias previas individuales y grupales.

Los talleres de construcción participativa desarrollados en la fase de planeación permitieron retroalimentar al equipo de investigación de los resultados de la fase de diagnóstico; definir y diseñar las acciones contenidas en la intervención; fortalecer las capacidades de todos los implementadores; consolidar las alianzas con las instituciones educativas y comunitarias involucradas en el programa; movilizar los recursos humanos y materiales necesarios; diseñar y validar materiales de educación y comunicación en diferentes soportes, por solo mencionar algunos de los resultados. Cabe destacar que su principal contribución fue garantizar la puesta en marcha de la fase de ejecución.

Entre las acciones ejecutadas, tuvieron especial connotación las charlas educativas realizadas en cada uno de los centros encuestados. En estas se abordaron temáticas como la bioecología básica de los mosquitos (con énfasis en su ciclo de vida y sus criaderos); las enfermedades que estos son capaces 
de vehicular y sus consecuencias sanitarias; las medidas preventivas más eficientes en el entorno doméstico y pautas sobre ordenamiento ambiental; por citar algunas. Además, se realizaron diversas actividades como: debates a partir de la proyección de audiovisuales (Documental «Conociendo los mosquitos Aedes - Transmisores de arbovirus», producido por el Instituto Oswaldo Cruz - FIOCRUZ, Río de Janeiro, Brasil) o la colocación de carteles en centros educativos y otros puntos de interés municipal, con el objetivo de difundir el mensaje entre todos los jarabacoenses, no solo entre aquellos en edad escolar. Cabe destacar que la mayoría de las actividades fueron planteadas por docentes y alumnos.

El desarrollo de las acciones antes referidas y la aceptación de las mismas dentro de las instituciones educativas demostraron la importancia de ampliar los escenarios de capacitación, no solo a los estudiantes de enseñanza media, sino también a los de enseñanza básica y a los universitarios. En consecuencia, se desarrollaron cinco talleres de especialización con la colaboración de investigadores y profesores de universidades españolas, dirigidos a estudiantes de la Universidad Agroforestal Fernando Arturo de Meriño (UAFAM) que también funcionarían como replicadores de los contenidos en las comunidades de residencia y centros de enseñanza media de Jarabacoa. El primero se realizó en diciembre de 2016 con la colaboración de la Universidad de Zaragoza y abordó las temáticas relacionadas con "Enfermedades Vectoriales Emergentes y Reemergentes"; el segundo, realizado en marzo de 2018 con la colaboración de la Universidad de Valencia, se denominó "Aportación al conocimiento de los mosquitos y su control: anticiparse a la transmisión de enfermedades"; el tercero, titulado "Nuevas tendencias para el control de plagas en el ámbito agricola y sanitario", se desarrolló en noviembre de 2018, a cargo de un investigador de NEIKER; el cuarto, también con la colaboración de la Universidad de Valencia, se realizó en febrero de 2019 bajo el nombre "Calidad del agua y biodiversidad: un caso práctico"; y el quinto, titulado "El control de plagas y la sanidad ambiental: un sector profesional de futuro", en colaboración con Laboratorios LOKÍMIKA S.A. en julio de 2019. Estos talleres contaron con una gran acogida académica y una afluencia de público, alrededor de los treinta participantes en cada uno. Conjuntamente, se llevaron a cabo charlas en dos colegios de enseñanza básica, a alumnos menores de diez años, en las que, mediante demostraciones, juegos de roles y confección de dibujos y carteles, se impartieron contenidos sobre prevención y control de enfermedades vehiculadas por mosquitos. Asimismo, se presentaron seis comunicaciones científicas en congresos nacionales e internacionales con las actualizaciones del trabajo desarrollado dentro el programa educativo.

Aunado a lo anterior, desde mediados de 2017 se creó una página de Facebook (@sacalelospiesalmosquito), en la cual el equipo de investigación informa con regularidad sobre las actividades que se llevan a cabo en el municipio y los eventos científicos en los que se participa. Cuenta con seguidores, principalmente, de República Dominicana, pero también Cuba, México, Guatemala, EE. UU., Brasil, Chile, España, Escocia y Letonia, entre otros. Asimismo, la página refleja noticias de interés nacional que implican a los vectores y/o las enfermedades que vehiculan. En 2018 se creó también una página web con esta misma temática (http:// sacalelospiesalmosquito.org/), que constituye otro recurso de Internet para ampliar la divulgación de nuestras acciones.

El monitoreo y la evaluación parcial de las acciones desarrolladas demostró la influencia de las mismas, tanto en la motivación de los escolares como en la participación de estos en las acciones promovidas por el programa. De igual forma se pudo apreciar una mayor movilización, tanto al interior de las escuelas como en las comunidades de residencia vinculadas a estos centros educacionales, en acciones de saneamiento y eliminación de riesgos asociados a la presencia de mosquitos. Asimismo, el monitoreo de los sitios de divulgación científica demostró un 
incremento de los usuarios de los mismos, así como del número de visitas y lecturas de las publicaciones. Además, en sentido general, se fortalecieron las capacidades de educadores y estudiantes para promover los conocimientos adquiridos, convirtiéndose en replicadores de estos contenidos y gestores de buenas prácticas de prevención y control.

\section{Discusión}

El programa educativo "Sácale los pies al mosquito», iniciativa pionera no solo en Jarabacoa, sino en República Dominicana, permitió articular paralelamente las investigaciones entomológicas de campo (Rodríguez-Sosa, Vásquez-Bautista, Fimia-Duarte, Guerrero y Alarcón-Elbal, 2018; Rueda, Rodríguez Sosa, Vásquez Bautista, Guerrero y Alarcón-Elbal, 2018; Borge de Prada, Rodríguez Sosa, Vásquez Bautista, Guerrero y Alarcón-Elbal, 2018; González et al., 2019; Rodríguez Sosa et al., 2019; Diéguez Fernández, Borge de Prada, Rodríguez Sosa, Vásquez Bautista y Alarcón-Elbal, 2019; Rodríguez Sosa, Diéguez Fernández, Borge de Prada, Vásquez Bautista y Alarcón-Elbal, 2019) con las acciones de educación y comunicación en el ámbito comunitario. De igual forma, se tuvieron en cuenta los contextos históricos y culturales, así como los cambios a nivel biológicos y psicológicos de los adolescentes, y las interacciones interpersonales y grupales (Pereira, 2011), elementos claves para el abordaje multidisciplinar de los escolares desde el diagnóstico, la planeación e implementación del programa educativo. Lo anterior contribuyó sobremanera a la contextualización de las acciones en el municipio cibaeño, tanto en relación con las particularidades del grupo de estudio como a los riesgos relacionados a estas enfermedades y sus vectores.

Las encuestas CAP han constituido, en las últimas décadas, una valiosa herramienta para recolectar información de forma fácil y rápida sobre un conjunto de indicadores, previamente establecidos por los equipos de investigación. La aplicación de este instrumento en el campo de las enfermedades vehiculadas por vectores ha permitido ofrecer recomendaciones para el diseño e implementación de estrategias comunitarias en diversos escenarios. Por tanto, los resultados obtenidos han contribuido no solo a aportar evidencias sobre el comportamiento de las variables e indicadores explorados, sino también a promover cambios positivos en el control vectorial y en la prevención de las enfermedades en las Américas, especialmente en relación con las arbovirosis y la malaria (OPS, 2008; Griffith et al., 2015; Delcid Morazán, Barcan Batchvaroff, González y Barahona Andrade, 2017; Hernández, 2017; Castañeda Porras y Zuleta Dueñas, 2018; Hernández, Pérez, Portal, Polo y Castro, 2019; López, Torres, Corominas Díaz, Guzmán Pérez y López Martínez, 2019).

El instrumento diseńado, validado y aplicado como parte del presente estudio, constituyó la primera encuesta CAP realizada en centros de enseńanza media en Jarabacoa. Dicha encuesta abarcó un amplio abanico de cuestiones, siempre con la problemática de los mosquitos como elemento central. Su aplicación contribuyó a articular, de una manera dinámica y congruente, los resultados del diagnóstico con el resto de las fases del programa educativo. El comportamiento de las variables sociodemográficas de los encuestados (sexo, edad, grado) fue representativo de la población estudiada, lo que permitió que las acciones educativas desarrolladas no solo satisficieran las necesidades de aprendizaje de los encuestados, sino también del resto de los escolares que se beneficiaron de la intervención.

La identificación de la falta de higiene, los depósitos con agua y la presencia de charcos, ríos y pantanos como principales factores de riesgo para la proliferación de mosquitos coincide con lo reportado en otros estudios similares (Castro et al., 2010; Castro et al., 2013; Hernández, Pérez, Portal, Polo y Castro, 2019). De igual forma, los sitios de cría más conocidos se corresponden con los identificados por Diéguez Fernández et al., (2019) en este propio municipio, lo que evidencia que estos 
conocimientos se asocian a los mensajes tradicionales al respecto y a los riesgos identificados en sus comunidades de residencia. Sin embargo, teniendo en cuenta que la intervención abarcó varios mosquitos de importancia médica con características ecológicas diferentes (Rodríguez Sosa et al., 2019), este constituyó uno de los temas más controversiales en la intervención.

El conocimiento preponderante de las arbovirosis con relación a otras enfermedades estudiadas, como la malaria o la filariasis linfática, se corresponde con la incidencia y distribución regional de estas (Alarcón-Elbal et al., 2017), así como con la cobertura mediática nacional. De hecho, en las últimas décadas se ha observado un aumento significativo de brotes epidémicos causados por enfermedades emergentes y reemergentes en República Dominicana, con implicaciones sanitarias en ocasiones subestimadas (Freitas, Alarcón-Elbal, Paulino-Ramírez y Donalisio, 2018). En relación con la cobertura mediática, cabe destacar que, tanto en el presente estudio como en otros similares, la televisión constituye la principal fuente de información debido a la gran variedad y cantidad de productos comunicativos promovidos en este medio (Hernández, 2017; Hernández et al., 2019; López et al., 2019).

A pesar de que la mayoría de los encuestados identificaron la picadura de mosquito como principal vía de transmisión de estas enfermedades, persisten criterios erróneos y en algunos casos hicieron referencia a factores de riesgo como vías de transmisión, lo que coincide con estudios similares realizados en países de Latinoamérica (Griffith et al., 2015; Delcid Morazán et al., 2017; Hernández, 2017; Hernández et al., 2019; López et al., 2019).

En uno de los estudios antes mencionados, realizado en Honduras, se pudo apreciar que aunque más del $90 \%$ de los encuestados ha escuchado hablar de las arbovirosis y refiere que estas son vehiculadas por mosquitos, menos del $40 \%$ las identifica entre las enfermedades promovidas por mosquitos del género Aedes, y algunos mencionaron la malaria entre estas (Delcid Morazán et al., 2017). En una zona urbana de Colombia, más del $90 \%$ conocían sobre el dengue y más del $35 \%$ había escuchado sobre fiebre amarilla y malaria, aunque la encefalitis estuvo entre las menos mencionadas (Criollo Fonseca, Bernal Barón y Castañeda Porras, 2014). En otro estudio similar realizado en una zona rural de ese mismo país, las arbovirosis fueron conocidas por menos del $20 \%$ de la población encuestada, y la malaria y la encefalitis por menos del $10 \%$ (Castañeda Porras y Zuleta Dueñas, 2018). En Cuba, más del $80 \%$ ha escuchado hablar de las arbovirosis y reconoció la picadura de mosquito como vía de transmisión, y la mayoría incluso hizo referencia a la picadura de mosquito infectado (Hernández et al., 2019). Resultados similares se encontraron en una comunidad de la provincia Peravia de República Dominicana donde, aunque solo se abordaron cuestiones relativas a Zika, el $79,4 \%$ manifestó conocer sobre la enfermedad y el $66,96 \%$ identificó al mosquito como vía de transmisión (López et al., 2019).

Las diferencias encontradas no solo entre los países, sino al interior de estos, unido a los resultados obtenidos en el presente estudio, refuerzan la importancia de contextualizar las acciones de comunicación y participación con las particularidades de cada momento y escenario entomológico y epidemiológico (Hernández, Pérez, Portal, Polo y Castro, 2019). Unido a lo anterior, no se debe subestimar la importancia de las encefalitis virales, la malaria o la filariasis linfática, debido a la elevada diversidad de especies de mosquitos de importancia médica que existen en el municipio (Rodríguez Sosa et al., 2019). En relación con la malaria, y a pesar de encontrarse en vías de erradicación en La Española, la enfermedad parece haber repuntado en los últimos meses (MSP/DIGEPI, 2019); por su parte, la filariasis linfática, parasitosis altamente incapacitante, también se encuentra en vías de erradicación en la isla, pero todavía son varios los desafíos respecto a su control (Gonzales et al., 2019). Por consiguiente, a partir del diagnóstico y de la incidencia real y 
potencial de estas enfermedades en el país, consolidar la percepción de riesgo, así como los puntos de contacto y diferencias entre estas en cuanto a vías de transmisión, sintomatología, incidencia, y medidas de prevención y control, por solo mencionar algunos elementos, constituyeron algunas de las prioridades de las acciones de educación y comunicación desarrolladas.

El hallazgo de que la mayoría de los escolares percibiera el riesgo de contraer alguna de estas enfermedades favoreció la motivación y participación de los mismos en la intervención, así como la asimilación de los contenidos. Lo anterior, a su vez, se correspondió con la actitud positiva de los escolares hacia el desarrollo de acciones de educación y comunicación para la prevención y el control de estas enfermedades, aun cuando la mayoría de ellos no tenían experiencias previas similares. Una encuesta recientemente aplicada en los municipios dominicanos de Bajos de Haina, Santo Domingo Norte y Miches reflejó que la mayoría percibe el riesgo a enfermar, aunque cabe destacar que en dicho estudio solo se exploró lo relativo al Zika (WVI/ OPS, 2016).

Por otra parte, la variedad de prácticas de prevención y control mencionadas coincide, en sentido general, con varios de los estudios anteriores. Sin embargo, las diferencias en cuanto al predominio y referencia de las mismas pudieran estar asociadas a las enfermedades exploradas, a las acciones promovidas en las estrategias de comunicación desarrolladas, así como a los principales factores de riesgo identificados en estos territorios. En Cuba y Honduras, al igual que en el presente estudio, se apreció un predominio de las medidas asociadas a la higiene de las viviendas y los patios y prevalecen las acciones individuales sobre las colectivas (Castro et al., 2010; Delcid Morazán et al., 2017; Hernández, et al., 2019). En tanto, en Colombia la destrucción de recipientes y criaderos de mosquitos fueron las medidas de control más conocidas y utilizadas (Criollo Fonseca et al., 2014). Sin embargo, en Panamá, al igual que en nuestro estudio, estas últimas estuvieron entre las menos mencionadas (Griffith et al., 2015).

El uso de las nuevas tecnologías, tanto para el desarrollo de los talleres como para la creación de las páginas web y Facebook, se correspondió con el elevado porcentaje de encuestados que manifestaron su interés porque existieran espacios y materiales digitales que divulgaran información sobre estas enfermedades y sus vectores, así como por el reconocimiento de Internet y las redes sociales como tercera fuente de información. Además, se tuvo en cuenta que las redes sociales funcionan para los adolescentes como un medio de socialización naturalizado en el que se definen espacios grupales $\mathrm{y}$ colectivos que fortalecen los vínculos sociales y motivación por ciertas temáticas (Bolis, 2015). En tanto, se facilita la participación, rompiendo los esquemas tradicionales de las acciones de comunicación en este campo. Por ello, las acciones de educación y comunicación permitieron, mediante mensajes claros, sencillos y oportunos, informar, sensibilizar y movilizar a los escolares en las acciones de prevención y control promovidas por el programa. En consecuencia, se pudo apreciar una gran aceptación de los espacios propuestos, así como una mayor empatía y retroalimentación entre los investigadores y sus audiencias.

Los resultados parciales, obtenidos a partir de la implementación del programa, demostraron que la escuela no solo constituye un espacio de socialización por excelencia, sino que, en colaboración con la familia y otras instituciones sociales y comunitarias, resulta de gran valor para promover conocimientos, actitudes y prácticas que contribuyen al control de los mosquitos, sobre todo en el ámbito domiciliar, y a la prevención de las enfermedades. Asimismo, garantiza una mayor sostenibilidad de las acciones permite la adecuación de las mismas a las particularidades de cada centro educativo y de las comunidades de residencia a las que prestan servicio, facilita el monitoreo y la evaluación a corto, mediano y largo plazo del programa, y permite involucrar a otros actores sociales. 
A pesar de lo anterior, las lecciones aprendidas desde las diferentes fases del programa pusieron al descubierto un conjunto de desafíos, entre los cuales caben destacar aquellos en los que, desde su identificación, ha estado trabajando el equipo de investigación: visibilizar este tipo de iniciativas tanto en la comunidad científica como en la sociedad en general; aportar evidencias científicas que contribuyan a su implementación en otras localidades del país; promover la movilización de recursos humanos y materiales que garanticen la sostenibilidad en el tiempo del programa; y generar nuevos espacios de divulgación científica que contribuyan a promover conocimientos, actitudes y prácticas adecuadas para la prevención y el control de las enfermedades vehiculadas por mosquitos no solo en los centros de enseñanza media, sino también en otras instituciones educativas.

Para finalizar, es evidente que la prevención debe ser la punta de lanza contra estas enfermedades, pues además de evitar el sufrimiento, las estimaciones económicas establecen que la prevención es monetariamente rentable a largo plazo. La reducción de la incidencia de estas enfermedades dependerá de la aplicación de medidas eficaces de lucha contra el mosquito, pero exige, forzosamente, el perfecto funcionamiento del engranaje formado no solo por la concientización social, sino también por la voluntad política, la intersectorialidad y multidisciplinariedad, el fomento de la investigación transdisciplinaria, la sostenibilidad medioambiental, el fortalecimiento de la legislación sanitaria y la lucha contra las desigualdades sociales (Alarcón-Elbal, 2016; Alarcón-Elbal, 2018). En este punto, no hay que olvidar que entre las principales causas que explican el fracaso de algunos de estos programas se encuentran la frágil continuidad de las actividades preventivas que compiten con la urgencia de otras demandas ambientales, sociales o sanitarias que constituyen para la ciudadanía, a priori, problemas de mayor peso que la eliminación de los criaderos de mosquitos (Schweigmann et al., 2009).

\section{Conflicto de interés}

Todos los autores que firman este manuscrito han ameritado con trabajo, esfuerzo y dedicación su inclusión en el mismo; y manifiestan la no existencia de conflicto de interés.

\section{Agradecimientos}

Los autores desean agradecer encarecidamente la colaboración de las siguientes instituciones educativas jarabacoenses: Centro Educativo Arcadio Antonio Tiburcio, Centro Educativo Profesora Eligia Violeta Tiburcio Guzmán, Centro Educativo Carlos Manuel Tiburcio Guzmán, Centro Educativo Hato Viejo, Colegio San José, Centro Educativo Profesora Isolina María Cruz, Colegio Nuestra Señora de La Altagracia, Liceo Secundario María Auxiliadora, Centro Educativo ANIJA (Colegio Cristiano Ayuda del Niño de Jarabacoa), Liceo Técnico Luis Ernesto Gómez Uribe, Doulos Discovery School, Centro Educativo de Formación Integral Cristiano (CEFIC), Escuela Técnica Salesiana Santo Domingo Savio, Centro Educativo José Antonio Guzmán Fabián, Colegio Experimental Fernando Arturo de Merińo (CEFAM), Centro Educativo Manuel Ubaldo Gómez y Centro Educativo Nieves María Valerio, así como al Centro Educativo Dulce María Tiburcio y al Centro Educativo Maximino Gutiérrez de la Cruz en los que también se realizaron charlas educativas para alumnos menores de diez años. Los autores también desean agradecer al Dr. Javier Lucientes (Universidad de Zaragoza, España), al Dr. Juan Rueda (Universidad de Valencia, España), al Dr. Mikel González (NEIKER, Espańa) y al Dr. Francisco Alberto Chordá por su colaboración en la realización de los cursos de especialización, así como a los compañeros de la UAFAM que prestaron apoyo puntual al proyecto: Kelvin Guerrero, Miguel Borge, Gerly Tiburcio y Samuel Aguilar. Por último, agradecer al Rancho Baiguate su apoyo constante e implicación oficial en el proyecto. 


\section{Referencias}

Alarcón-Elbal, P. M. (2016). El dengue, la ineludible necesidad de invertir en prevención. Listín Diario. Recuperado de https://listindiario.com/economia/2016/01/04/402545/el-dengue-la-ineludible-necesidad-de-invertir-en-prevencion

Alarcón-Elbal, P. M. (2018). Deforestation and mosquito-borne diseases: another 'wake-up call' to Latin America. InterAmerican Journal of Medicine and Health, 1(1), e201801003. Recuperado de https://iajmh.com/iajmh/article/view/23/27

Alarcón-Elbal, P. M., Paulino-Ramírez, R., DiéguezFernández, L., Fimia-Duarte, R., Guerrero, K. A. \& González, M. (2017). Arbovirosis transmitidas por mosquitos (Diptera: Culicidae) en la República Dominicana: una revisión. The Biologist (Lima), 15(1), 193-219. Recuperado de http://revistas.unfv.edu.pe/index.php/rtb/ article/view/155/147

Bolis, N. (2015). Adolescentes y socialización en espacios virtuales. VII Congreso Internacional de Investigación y Práctica Profesional en Psicologia, XXII Jornadas de Investigación y XI Encuentro de Investigadores en Psicología del MERCOSUR. Facultad de Psicología, Universidad de Buenos Aires, Argentina. Recuperado de https://www. aacademica.org/000-015/423.pdf

Borge de Prada, M., Rodríguez Sosa, M. A., Vásquez Bautista, Y. E., Guerrero, K. A. \& Alarcón-Elbal, P. M. (2018). Mosquitos (Diptera, Culicidae) de importancia médica asociados a residuos sólidos urbanos en Jarabacoa, República Dominicana. Revista Salud Jalisco, (Número Especial), 20-27. Recuperado de https://www.medigraphic.com/ pdfs/saljalisco/sj-2018/sj18Ed.pdf

Casas Anguita, J., Repullo Labradora, J. R. \& Donado Campos, J. (2003). La encuesta como técnica de investigación. Elaboración de cuestionarios y tratamiento estadístico de los datos (I). Atención Primaria, 31(8), 527-538. Recuperado de http://www.unidaddocentemfyclaspalmas. org.es/s/9+Aten+Primaria+2003.+La+Encuesta $+\mathrm{I} .+$ Custionario $+\mathrm{y}+$ Estadistica.pdf
Castañeda Porras, O. \& Zuleta Dueñas, L. P. (2018). Conocimientos, actitudes y prácticas para el control de enfermedades transmitidas por vectores en zona rural dispersa, San Luis de Palenque, Casanare-Colombia, 2017. Revista Médica de Risaralda, 24(2), 108-114. Recuperado de http:// www.scielo.org.co/pdf/rmri/v24n2/0122-0667rmri-24-02-108.pdf

Castrillón Castro, A., Santa Gil, V., Loaiza Giraldo, D., Ortiz Garcés, D., \& Aragon Múnera, V. (2017). Relación entre conocimientos, hábitos y participación comunitaria frente a la transmisión del dengue y chikungunya. CES Salud Pública, 8(1), 70-81. Recuperado de http://revistas.ces. edu.co/index.php/ces_salud_publica/article/ view/4422

Castro, M., Gálvez, C., Sánchez, L., Pérez, D., Polo, V., Concepción, D., Sebrango, C. \& Van der Stuyft. P. (2010). Encuesta poblacional sobre conocimientos y percepciones acerca de dengue contra prácticas preventivas en el municipio Lisa. Revista Cubana de Medicina Tropical, 62, 245-253. Recuperado de http://scielo.sld.cu/pdf/ $\mathrm{mtr} / \mathrm{v} 62 \mathrm{n} 3 / \mathrm{mtr} 13310 . \mathrm{pdf}$

Castro, M., Sánchez, L., Pérez, D., Sebrango, C., Shkedy, Z. \& Van der Stuyft, P. (2013). The relationship between economic status, knowledge on dengue, risk perceptions and practices. PLOS ONE, 8(12), e81875. Recuperado de https:// journals.plos.org/plosone/article?id=10.1371/ journal.pone.0081875

Criollo Fonseca, I. Z., Bernal Barón, A. Y. \& Castañeda Porras, O. (2014). Conocimientos, actitudes y prácticas sobre dengue, tras aplicación de estrategias de movilización social. Yopal-Casanare, Colombia, 2012. Investigaciones Andina, 16(29), 1001-1015. Recuperado de http://www.scielo. org.co/pdf/inan/v16n29/v16n29a02.pdf

Delcid Morazán, F. A., Barcan Batchvaroff, M. E., González, C. H. \& Barahona Andrade, D. S. (2017). Conocimientos, actitudes y prácticas sobre las arbovirosis. Archivos de Medicina, 13(1), 5 Recuperado de http://www.archivosdemedicina.com/medicina-de-familia/ 
conocimientos-actitudes-y-praacutecticas-sobre-las-arbovirosis.pdf

Diéguez Fernández, L., Borge de Prada, M., Rodríguez Sosa, M. A., Vásquez Bautista, Y. E. \& Alarcón-Elbal, P. M. (2019). Un acercamiento al conocimiento de los hábitats larvarios de Aedes (Stegomyia) aegypti (Diptera: Culicidae) en el entorno doméstico en Jarabacoa, República Dominicana. Revista Cubana de Medicina Tropical, 71(3), en prensa.

Diéguez Fernández, L., Sosa Cabrera, I. \& Pérez Arruti, A. E. (2013). La impostergable participación comunitaria en la lucha contra el dengue. Revista Cubana de Medicina Tropical, 65(2), 272-276. Recuperado de http://scielo. sld.cu/pdf/mtr/v65n2/mtr15213.pdf

Freitas, A. R. R., Alarcón-Elbal, P. M., PaulinoRamírez, R. \& Donalisio, M. R. (2018). Excess mortality profile during the Asian genotype chikungunya epidemic in the Dominican Republic, 2014. Transactions of the Royal Society of Tropical Medicine and Hygiene, 112(10), 443-449. Recuperado de https://www.ncbi. nlm.nih.gov/pubmed/30085307

Gonzales, M., Baker, M. C., Celestino, A., Santa Morillo, D., Chambliss, A., Adams, S., Gyapong, M. \& Kyelem, D. (2019). How lymphatic filariasis was eliminated from an urban poor setting in Santo Domingo, Dominican Republic. International Health, 11(2), 108-118. Recuperado de https:/www.ncbi.nlm.nih.gov/ pubmed/30285112

González, M. A., Rodríguez Sosa, M. A., Vásquez Bautista, Y. E., Diéguez Fernández, L., Borge de Prada, M., Guerrero, K. A. \& Alarcón-Elbal, P. M. (2019). Micro-environmental features associated to container-dwelling mosquitoes (Diptera: Culicidae) in an urban cemetery of the Dominican Republic. Revista de Biología Tropical, 67(1), 132-145. Recuperado de https://revistas.ucr.ac.cr/index.php/rbt/article/ view/33158/36940
Griffith, M., Rovira, J., Torres, R., Calzada, J., Victoria, C. \& Cáceres, L. (2015). Conocimientos, actitudes y prácticas sobre la malaria en la población indígena guna de la comarca de Madungandí, Panamá, 2012. Biomédica, 35, 480-495. Recuperado de http://www.scielo. org.co/pdf/bio/v35n4/v35n4a06.pdf

Hernández, Y. (2017). Gestión de comunicación comunitaria para la prevención de arbovirosis. Propuestas teórico-metodológicas y prácticas para su fortalecimiento en el contexto cubano (tesis de Maestría). Facultad de Comunicación Social, Universidad de La Habana. La Habana, Cuba.

Hernández, Y., Pérez, D., Portal, R., Polo, V. \& Castro, M. (2019). Abordaje de la comunicación para la prevención de arbovirosis en Cuba versus conocimientos, percepciones y prácticas de la población. Revista Cubana de Medicina Tropical, 71(3), en prensa.

López, E., Torres, D. Corominas Díaz, A. V., Guzmán Pérez, L. M. \& López Martínez, L. J. (2019). Conocimientos, aptitudes y prácticas acerca de la infección por el virus del Zika en los residentes de la Bahía de las Calderas, provincia Peravia, República Dominicana, en el período febrero-abril 2016. Ciencia y Salud, 3(1), 61-67. Recuperado de https://revistas.intec.edu.do/index. $\mathrm{php} / \mathrm{cisa} /$ article/view/1332/1695

Ministerio de Salud Pública/Dirección General de Epidemiología. (2019). Boletín Epidemiológico Semana. Semana Epidemiológica (SE) (25). Recuperado de http://digepisalud.gob.do/docs/ Boletines\%20epidemiologicos/Boletines\%20 semanales/2019/Boletin\%20Semanal\%20252019.pdf

Organización Mundial de la Salud. (2017). Respuesta mundial para el control de vectores 2017-2030. Documento de contexto para informar las deliberaciones de la Asamblea Mundial de la Salud en su 70. ${ }^{a}$ reunión. Versión 5.4. Ginebra: OMS. Recuperado de https://www.who.int/malaria/ areas/vector_control/Draft-WHO-GVCR2017-2030-esp.pdf 
Organización PanamericanadeSalud.(2008).Encuesta sobre Conocimientos, Actitudes y Prácticas (CAP): Una herramienta para el abordaje intercultural de la malaria. Recuperado de https://www.paho. org/pan/index.php?option=com_docman\&view=download\&alias=73-encuesta-sobre-conocimientos-actitudes-y-practicas-cap-abordaje-intercultural-de-la-malaria\&category_ slug=publications \&Itemid $=224$

Organización Panamericana de Salud. (2016). Estrategia para la prevención y el control de las enfermedades arbovirales. 55. Consejo Directivo 68. a Sesión del Comité Regional de la OMS para Las Américas. Washington, D.C: OPS. Recuperado de http://iris.paho.org/ xmlui/bitstream/handle/123456789/34486/ CD55-FR-s.pdf;jsessionid=F2F34B72B47491280748015D13E397A6? sequence $=2$

Orgaz Agüera, F. \& Cañero Morales, P. (2015). El ecoturismo como motor de desarrollo en zonas rurales: un estudio de caso en República Dominicana. Ciencia y Sociedad, 40(1), 47-76. Recuperado de https://revistas.intec.edu.do/index.php/ciso/ article/view/1007/pdf-OrgazAguera

Pereira, R. (2011). Adolescentes en el siglo XXI. Entre impotencia, resiliencia y poder. Madrid, España: Morata.

Rodríguez-Sosa, M. A., Vásquez-Bautista, Y. E., Fimia-Duarte, R., Guerrero, K. A. \& AlarcónElbal, P. M. (2018). Primer registro de Uranotaenia cooki (Diptera: Culicidae) para República Dominicana. Revista Colombiana de Entomología, 44(1), 141-144. Recuperado de http://www.scielo.org.co/pdf/rcen/v44n1/ 0120-0488-rcen-44-01-00141.pdf

Rodríguez Sosa, M. A., Rueda, J., Vásquez Bautista, Y. E., Fimia-Duarte, R., Borge de Prada, M., Guerrero, K. A. \&Alarcón-Elbal, P. M. (2019). Diversidad de mosquitos (Diptera: Culicidae) de Jarabacoa, República Dominicana. Graellsia, 75(1), e084. Recuperado de http://graellsia. revistas.csic.es/index.php/graellsia/article/ view/591/771
Rodríguez Sosa, M. A., Diéguez Fernández, L., Borge de Prada, M., Vásquez Bautista, Y. E. \& Alarcón-Elbal, P. M. (2019). Sitios de cría de Aedes albopictus (Skuse) (Diptera: Culicidae) en el entorno doméstico en Jarabacoa, República Dominicana. Revista Chilena de Entomología, 45(3): 403-410. Recuperado de http://www. insectachile.cl/rchen/pdfs/2019v45-3/ Rodr\%C3\%ADguez-Soza_et_al_2019.pdf

Rueda, J., Rodríguez Sosa, M. A., Vásquez Bautista, Y. E., Guerrero, K. A. \& Alarcón-Elbal, P. M. (2018). Primera cita de Culex (Culex) garciai González Broche, 2000 (Diptera: Culicidae) para LaEspañola. Anales de Biología, 40, 95-101. Recuperado de https://digitum.um.es/digitum/ bitstream/10201/60900/1/40_2018_11.pdf

San Martín, J, \& Prado, M. (2004). Percepción del riesgo y estrategias de comunicación social sobre el dengue en las Américas. Revista Panamericana de Salud Pública, 15(2), 135-139. Recuperado de http://www.scielosp.org/scielo.php?script=sci_arttext\&pi$\mathrm{d}=$ S1020-49892004000200014

Sánchez Valdés, L. (2006). Proceso y resultados de la prevención comunitaria del dengue (tesis Doctoral). Institutode Medicina Tropical "Pedro Kouri", La Habana, Cuba. Recuperado de http://tesis.sld. $\mathrm{cu} /$ index.php? $\mathrm{P}=$ DownloadFile\&Id=321.

Sánchez, L., Pérez, D., Alfonso, L., Castro, M., Sánchez, L. M., Van der Stuyft, P. \& Kourí, G. (2008). Estrategia de educación popular para promover la participación comunitaria en la prevención del dengue en Cuba. Revista Panamericana de Salud Pública, 24(1), 61-69. Recuperado de https://www.scielosp.org/article/ssm/content/raw/?resource_ssm_path=/ media/assets/rpsp/v24n1/v24n1a08.pdf

Schweigmann, N., Rizzotti, A., Castiglia, G., Gribaudo, F., Marcos, E., Burroni, N., Freire, G., D’Onofrio, V., Oberlander, S., Schillaci, H., Gómez, S., Maldonado, S. \& Serrano, C. (2009). Información, conocimiento y percepción sobre el riesgo de contraer el dengue en Argentina: dos experiencias de intervención para 
generar estrategias locales de control. Cadernos de Saúde Pública, 25(Suppl. 1), S137-S148. Recuperado de http://www.scielo.br/pdf/csp/ v25s1/13.pdf

World Health Organization. (2012). Handbook for integrated Vector Management. Geneva: WHO. Recuperado de https://apps.who.int/iris/bitstream/handle/10665/44768/9789241502801_ eng.pdf;jsessionid=393D073D5C26A8FF1CEAA18701351DA5?sequence $=1$
WorldVision Internacional/Organización Panamericana delaSalud. (2016). EmergenciaZIKV:resultadosdel proceso de consulta conocimientos, actitudes y prácticas (CAP) sobre ZIKV. Informe de país: República Dominicana. Recuperado de https://www.paho. org/hq/dmdocuments/2016/2016-cha-informe-tecnico-cap-dor-es.pdf

\section{Datos de filiación}

Yohan Enmanuel Vásquez Bautista. Estudiante de Educación y Asistente de Investigación del Laboratorio de Entomología, Universidad Agroforestal Fernando Arturo de Merińo (UAFAM). Jarabacoa, República Dominicana. Asistente de Investigación del Laboratorio de Entomología de la UAFAM. Av. Norberto Tiburcio, No. 37, La Confluencia, 41000, Jarabacoa, La Vega.

Yisel Hernández Barrios. Investigadora y Profesora del Departamento Control de Vectores, Instituto de Medicina Tropical Pedro Kourí (IPK). La Habana, Cuba. Investigadora. Grupo de Comunicación Social; Departamento Control de Vectores; Centro de Investigaciones, Diagnóstico y Referencia (CIDR); Instituto de Medicina Tropical Pedro Kourí (IPK). Autopista Novia del Mediodía KM 6 1⁄2. La Lisa, La Habana, Cuba. CP 11400.

María Altagracia Rodríguez Sosa. Estudiante de Agronomía y Asistente de Investigación del Laboratorio de Entomología, Universidad Agroforestal Fernando Arturo de Meriño (UAFAM). Jarabacoa, República Dominicana. Asistente de Investigación del Laboratorio de Entomología de la UAFAM. Av. Norberto Tiburcio, No. 37, La Confluencia, 41000, Jarabacoa, La Vega.

Elizabeth del Carmen Rosario. Estudiante de Educación y Técnico del Laboratorio de Entomología, Universidad Agroforestal Fernando Arturo de Meriño (UAFAM). Jarabacoa, República Dominicana. Personal de Apoyo a la Investigación del Laboratorio de Entomología de la UAFAM. Av. Norberto Tiburcio, No. 37, La Confluencia, 41000, Jarabacoa, La Vega.

Jesús Confesor Durán Tiburcio. Estudiante de Agronomía y Técnico del Laboratorio de Entomología, Universidad Agroforestal Fernando Arturo de Meriño (UAFAM). Jarabacoa, República Dominicana. Personal de Apoyo a la Investigación del Laboratorio de Entomología de la UAFAM. Av. Norberto Tiburcio, No. 37, La Confluencia, 41000, Jarabacoa, La Vega.

Pedro María Alarcón-Elbal. Investigador y Profesor del Laboratorio de Entomología, Universidad Agroforestal Fernando Arturo de Merińo (UAFAM). Jarabacoa, República Dominicana. Personal de Apoyo a la Investigación del Laboratorio de Entomología de la UAFAM. Av. Norberto Tiburcio, No. 37, La Confluencia, 41000, Jarabacoa, La Vega. 